\title{
IoT: Electrocardiogram (ECG) Monitoring System
}

\author{
Kristine Joyce P. Ortiz*, John Peter O. Davalos, Elora S. Eusebio, Dominic M. Tucay \\ Department of Computer Engineering, Malayan Colleges Laguna, Philippines
}

\begin{tabular}{l}
\hline Article Info \\
\hline Article history: \\
Received Dec 7, 2017 \\
Revised Jan 25, 2018 \\
Accepted Feb 20, 2018 \\
\hline
\end{tabular}

Keywords:

C\#

Electrocardiogram (ECG)

Internet of Things (IoT)

MATLAB

Rapid Application

Development (RAD)

\begin{abstract}
Internet of Things (IoT) has many applications in the medical field. With remote-information gathering, healthcare professionals can evaluate, diagnose and treat patients in remote locations using telecommunications technology. This study aimed to develop a small-scale electrocardiogram (ECG) monitoring device that will measure heart rates and waveforms and send the data in a database and a web server. An ECG acquisition device was developed using a single-lead heart rate monitor sensor and an Arduino microcontroller. A program, which will process, analyze and upload the ECG data is coded using MATLAB and C\# programs. The collected information is viewed in a Graphical User Interface (GUI) display, coded using C\# and in a webpage. Rapid Application Technology (RAD) was used in the methodology, which began with a quick design of the system. The hardware and software systems underwent a prototyping cycle for development. Once finished, the integration of the system is conducted to construct a complete IoT-based ECG monitoring system. For testing using t-test, a sample size of 18 and a $\alpha=0.05$ is used. Testing resulted into t-test values that lie in the non-critical zone for all ECG parameters, denoting that there is no significant difference between the gathered data. The device's percent reliability in detecting ECG conditions such as normal sinus rhythm, sinus tachycardia, sinus bradycardia and flatline, is $83.33 \%$. The percent difference for the heart rate is $0.35 \%$, which falls within the acceptable medical standard of $99 \%$ accuracy. The device was deemed functional and reliable.
\end{abstract}

Copyright $@ 2018$ Institute of Advanced Engineering and Science. All rights reserved.

\section{Corresponding Author:}

Kristine Joyce P. Ortiz,

Department of Computer Engineering,

Malayan Colleges Laguna,

Pulo-Diezmo Road, Cabuyao City, Laguna, 4025 Philippines

Email: kjportiz@mcl.edu.ph

\section{INTRODUCTION}

Common hospital set-up include nurses making rounds in each ward, checking each equipment, and then record the findings on paper takes a significant amount of time. Routine reviews and check-ups mean that possible irregularities in the vital signs can only be detected during each visit. The manual recording also makes the system vulnerable to errors attributed to human fault.

The study focuses on developing a small-scale heart monitoring system using Electrocardiogram (ECG) technology [1]. The device is composed of a single-lead heart rate monitor sensor which converts the ECG signal into analog data, and an Arduino microcontroller, which converts the analog data into ASCII. The data is sent to an external computer where it will be processed into ECG rates and waveforms by a MATLAB program. A C\# program will analyze the ECG data for abnormalities [2] such as Tachycardia where the beats per minute $(\mathrm{BPM})>100$, Bradycardia $(\mathrm{bpm}<100)$ and Asystole (flatline) and also display the collected information in a Graphical User Interface (GUI). The collected information are uploaded into a MySQL database and viewed in a webpage [3]. By then, the information becomes accessible to relevant people even in long-distance. The ECG monitoring system's IoT's functionality is limited within a specific organization such as a hospital. Internet connection is required to be able to access the collected information 
as the data are automatically uploaded to the database. Usage of single-lead ECG is not as accurate as the standard 12-leads ECG model resulting into not being able to give in-depth details about the heart's electrical activity. Single-lead was used due to unavailability of multiple leads ECG module in the country. During the ECG acquisition process, the patient must be static free, stripped of any metallic objects, lying down and stationary. Detecting abnormal conditions is dependent on the heart rate (HR) parameter only.

Some of the related literature concentrates on the data acquisition and the hardware design of the system. While majority uses wireless transmission such as General Packet Radio Service (GPRS), global system for mobile communications (GSM), and Internet for remote monitoring. The proposed design by Abo-Zahhad et.al. is capable of measuring vital signs such as heart-rate, blood pressure, blood oxygen level, and ECG of the patient and sending results using internet [4]. But for abnormal readings, alerts or data are sent through GPRS for the doctor and GSM for the patient's relatives to minimize the cost of sending data. The authors did not provide tests and results to prove that their proposed design is working properly. It is evident in their studies that majority of the telemedicine uses internet to transfer data, be it wired or wireless. The summarized table they provided shows that majority of authors concentrates on the hardware design, signal acquisition, and energy efficiency. It was not clearly stated whether the telemedicine devices are capable of giving diagnosis based on the acquired data. A device that also transmits data using GSM was developed by Patil et.al. The design uses a 3-lead ECG device and shows the results in Liquid Crystal Display (LCD) character display [5]. The paper written by Steinhubl et.al. used Bluetooth or frequency modulation of ultrasound to transfer the ECG measurement directly to the smartphone [6]. It uses a fingercontact on the back of the smartphone to activate the reading process and the results are transmitted to the doctor in portable document format (PDF) format. It can give a diagnosis unlike with the previous literature. However, it would take time for the doctor to see the results since the results will be converted first to PDF file before sending it to the doctor. Miao et.al.'s device uses Bluetooth to transfer data [7]. However, Miao et. al. designed their own context-aware ECG device which can be used while resting or moving. Major concern is the use of secure digital (SD) card to store data since it will be very limited based on the capacity of the card. The system does not provide a diagnosis on the patient. It only shows the readings of the different parameters. Another paper written by Irianto et. al. used 12-lead ECG, graphical LCD and computer for display of ECG, speakers to hear the heartbeats, and printer as an additional output. The authors designed the bioamplifiers for the ECG but it was not clearly stated if they were able to provide diagnosis based on the ECG readings [8]. The design by Rizal et.al. that measures vital signs such as blood pressure, pulse rate, ECG and temperature is similar to the study because that data is transferred wirelessly(doctor and nurse) and has GUI for the result. Based on the sample GUI, the diagnosis for the heart rate and the temperature are indicated but not the diagnosis for the ECG and blood pressure [9]. From these existing and previous studies, the proponents decided combine the use of internet and providing a possible diagnosis based on the ECG readings. Both the doctor and patient's relative can see the ECG readings and diagnosis. Only the doctor can see all the patients under his care and add comments on their conditions.

With Internet connected medical equipment, the devices are able to automatically relay collected information to healthcare professionals making the equipment more effective by allowing real-time monitoring of patient health. A data is shown in a complete detailed picture of patient status which can then be reviewed wherever the physician may be. By applying remote-patient monitoring, healthcare professionals can continuously gather data and use it to help diagnose the patient so they could receive the best treatment as quickly as possible. Some projects also aim to prevent or delay the need for expensive long-term care and reduce the need for unplanned hospital visits. Another benefit of using IoT in medical technology is to lower the margin of errors [3]. Detailed explanation on how the IoT: Electrocardiogram monitoring system was developed and tested will be discussed on the succeeding sections. Section 2 explains the methodology used and how the ECG monitoring device works. Section 3 shows the results of the testing and its interpretation. Section 4 shows the conlusion based on the results.

\section{RESEARCH METHOD}

Rapid Application Development (RAD) [10] begins with research and data gathering herein conducted as the review of the related literatures and canvassing of hardware components as shown in Figure 1. A quick design is built based on the theoretical framework, other related studies, and the availability of hardware components in the market. Afterwards, the researchers decided that the study would be based on the construction of a small-scale heart monitoring system utilizing a single-lead heart rate monitor sensor (DFRobot Heart Rate Monitor Sensor Gravity), microcontroller technology (DFRobot Arduino Uno R3), C\# (Visual Studio 2015) and MATLAB programming (MATLAB R2016a). System integration follows the development of the hardware (the connection of the individual components) and software system (coding of the MATLAB and C\# programs). The system will undergo prototyping. Afterwards, the prototype will 
undergo testing involving the comparison of the data gathered from the single-lead ECG device prototype and a Meca303i 12-lead ECG machine. Results will be evaluated using t-test, percent difference and percent reliability statistical tools and a conclusion will be formulated [11].

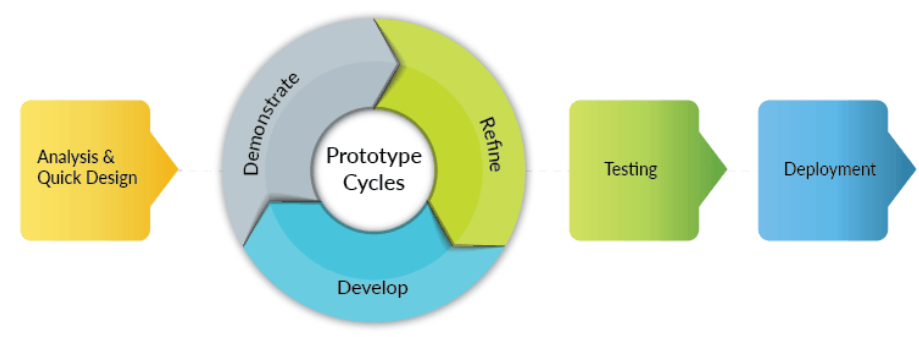

Figure 1. RAD Methodology Diagram (Wavemaker Solutions, 2016)

As shown in Figure 2, show the input-process-output flow of the system. The input will be the patient's heart activity read by the single-lead ECG acquisition device. The signal from the ECG acquisition device will be processed by the Arduino microcontroller. The results are displayed in the website and GUI of the device.
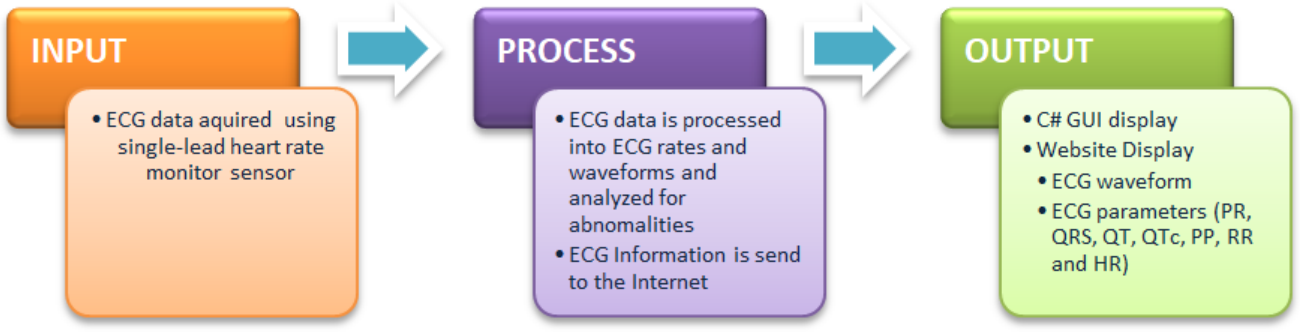

Figure 2. Input-Process-Output (IPO) Chart of ECG Monitoring Device

Illustrated in Figure 3 is the architecture for the fully integrated IoT: Electrocardiogram (ECG) monitoring system. The hardware and software components were combined to complete the ECG monitoring system. Transmission of data through Internet achieves the IoT part of the study.

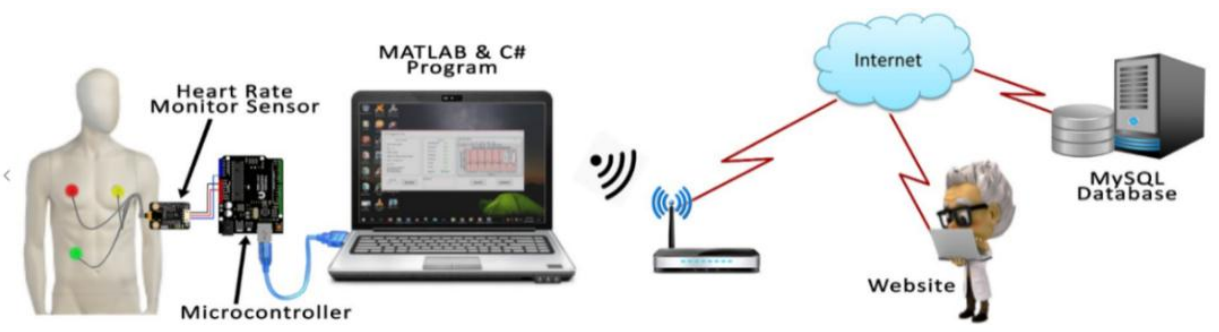

Figure 3. Architecture of the ECG Monitoring System

An Arduino program converts the analog data into ASCII data and sends it to an external computer using serial data transmission. A C\# program asks the user what module they want to run, the patient or the doctor module as shown in Figure 4. In the patient module, the program will ask for the patient ID and passkey and will get the communication (COM) port from wherein the microcontroller is connected. The program will retrieve the patient information from the database and display it on the C\# GUI. The program continuously read the data coming from the COM ports and analyze it until the user logs out or closes the 
program. If the data is valid, the program will pass it onto the MATLAB DLL program. The MATLAB DLL is separated into two parts, the ECG_PLOT program and the ANALYZER program. The ECG_PLOT takes the ASCII data and stores it into variables. The variables are plotted and extrapolated resulting in the ECG waveform graph and saves the file as .mat which is a MATLAB file as shown in Figure 5. The ANALYZER program shown in Figure 6 analyzes the saved file and identifies the following ECG parameters: PR, QRS, QT, QTC, PP, RR and the heart rate (HR). It then returns the collected data into the C\# program so that it can be displayed in the GUI. The collected information is uploaded into a MySQL database[4] and displayed in a webpage. Once the user logs out, the program will return into the user preference selection form. In the doctor module, the program will ask for the doctor ID and passkey. If both are correct, the patient information attributed to the doctor is downloaded from the MySQL database and arrange in a table in the C\# GUI as shown in Figure 7. The user will select an entry from the table. Upon selection, the .mat file of that patient will be downloaded from the web server. The user will be prompted analysis of the data if necessary. If yes, the MATLAB ECG_ANALYZER DLL will load and the ECG parameters will be displayed in the GUI. The C\# diagnostic program will also load and determine the sinus status of the ECG data and display it on the GUI. The user can choose to add a message or comment to the file, which will be uploaded into the MySQL database in the patient's record, and subsequently displayed in the webpage. The .mat file will be automatically deleted after its information are displayed in the C\# GUI, for security purposes and prevention of file duplication. Once the user logs out, the program will return into the user preference selection form.

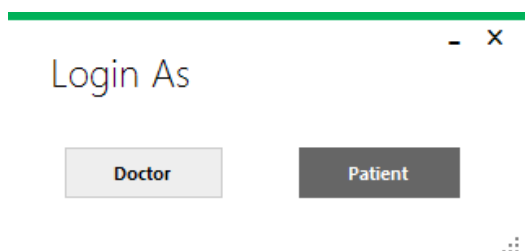

Figure 4. Login Page of the Website

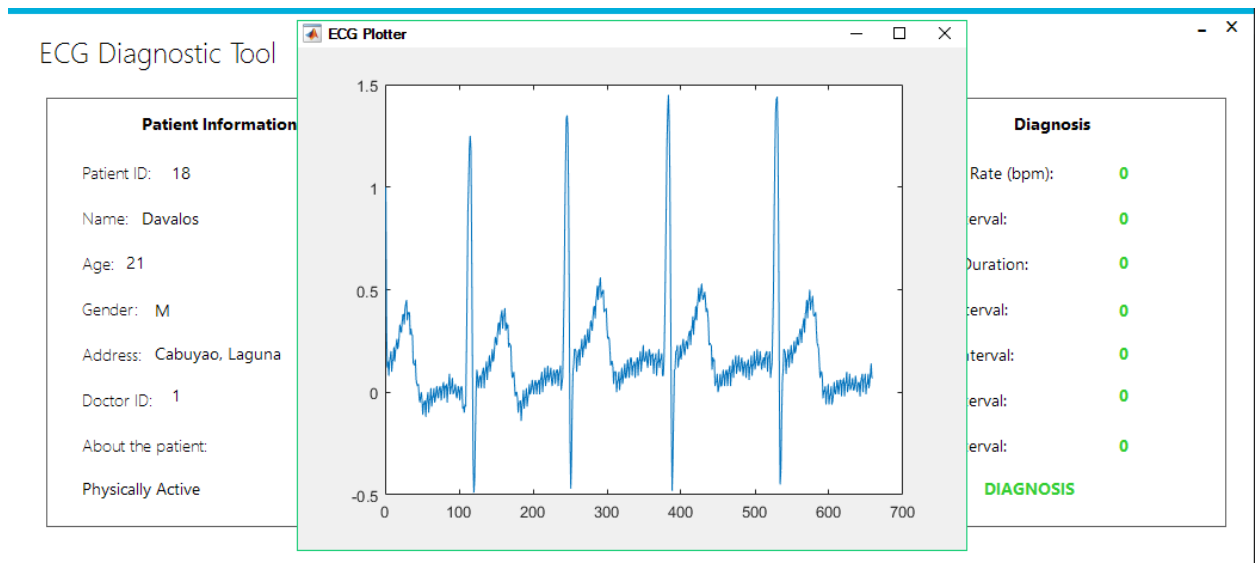

Figure 5. ECG Plotter using MATLAB 


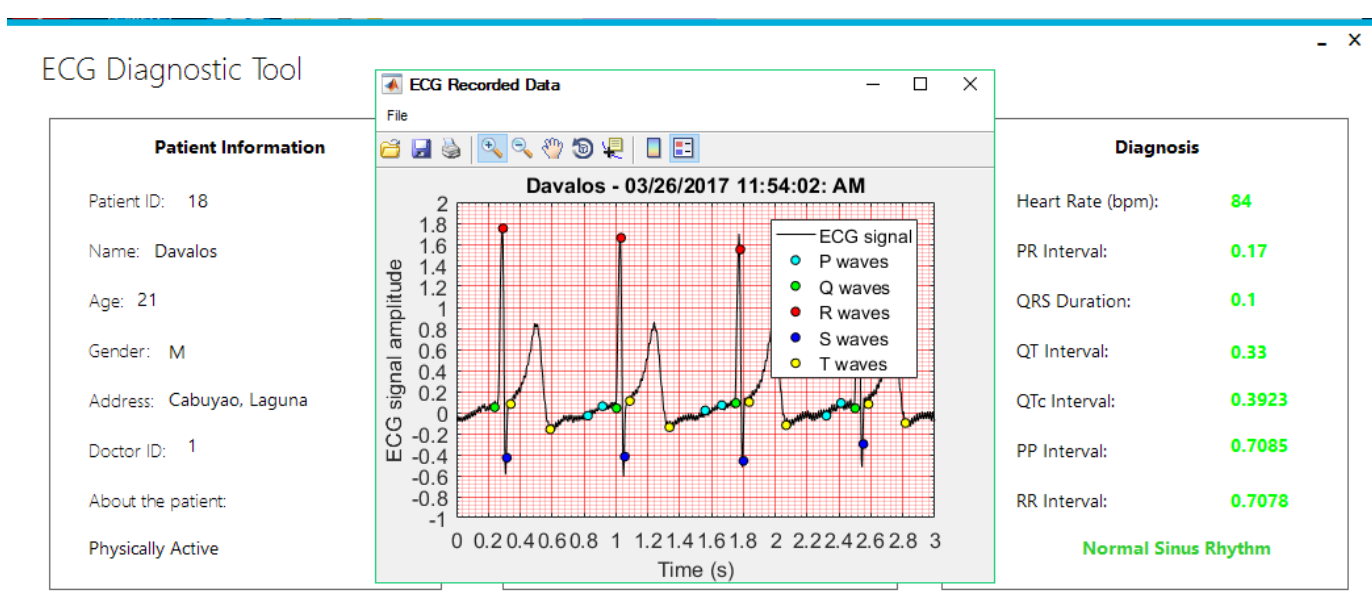

Figure 6. GUI of the Collected Data from ECG Analyzer Program

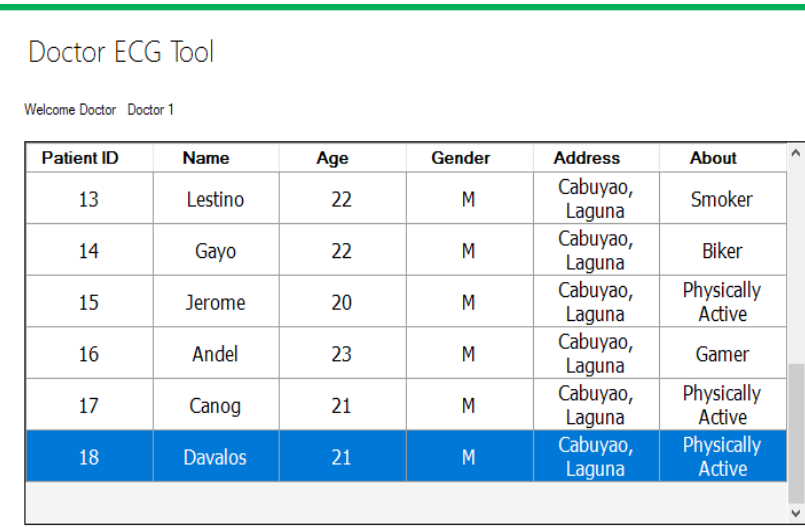

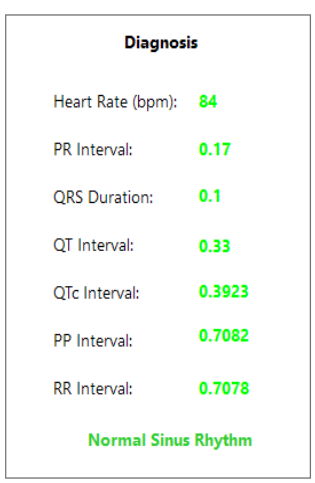

Message:

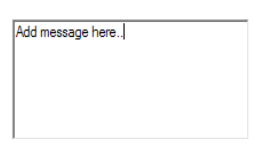

Send Messag

Figure 7. Doctor's ECG Tool

The performance of the device in generating heart rates and waveforms were tested against the readings from a Meca303i 12-lead ECG machine. Another experiment determines if the system was capable of detecting irregular ECG conditions such as Tachycardia, Bradycardia and Asystole (flatline). Statistical tools used were t-test with a sample size of 18 and $\alpha=0.05$ [12], percent difference and percent reliability. Alpha $(\alpha)$ value is the cut-off for identifying the significant difference of two sample sets [13]. T-test was used to determine if there is a significant difference between the two sample sets. This was used for comparison testing of ECG machine and device prototype results. The t-value was computed using Equation 1.

$$
t=\frac{\overline{\mathrm{X}}-\mu}{\frac{s}{\sqrt{n}}}
$$

where: $\overline{\mathrm{X}}=$ Sample Mean

$\mu=$ hypothesized population mean

$\mathrm{s}=$ sample standard deviation

$\mathrm{n}=$ sample size

Degrees of freedom $=\mathrm{n}-1$

Percent Difference (Equation 2) was used to determine the relative difference between two samples. For medical applications, the percent difference should be less than $1 \%$. 


$$
\% \text { difference }=\left|\frac{\text { Expected }- \text { Actual }}{\frac{\text { Expcted }+ \text { Actual }}{2}}\right| \times 100 \%
$$

where: $\quad$ Expected = ECG value gained from Meca303i 12-lead ECG machine Actual $=$ ECG value gained from ECG device prototype

Percent Reliability (Equation 3) was used to determine the overall reliability rate of the device from the test's experiments successful outcomes vs. the total number of outcomes.

$$
\% \text { reliability }=\frac{\text { number of successful attempts }}{\text { total number of attempts }} \times 100 \%
$$

\section{RESULTS AND ANALYSIS}

The ECG waveform is denoted by 5 major points, namely, P, Q, R, S and T. Figure 8 shows the ECG waveform points and the interval of major points. P wave is the arch before the QRS complex. Q wave is the first negative deflection of the QRS complex followed by $\mathrm{R}$ wave which is the positive deflection. S wave is the negative deflection after the $\mathrm{R}$ wave and $\mathrm{T}$ wave is the last arch after the QRS complex[14]. To perform a waveform comparison, each parameter was recorded from the actual ECG device and the ECG device prototype and a comparison of the results was conducted. The heart rate from the 12-lead ECG machine as shown in Figure 9 was tested against the heart rate from the single-lead ECG device prototype. Eighteen people were tested using a single-lead ECG device prototype as shown in Figure 10(a), and a 12lead ECG machine as shown in Figure 10 (b), generating 18 samples. The samples were gathered to conduct a small sample mean test, specifically a t-test. The t-test computations are conducted using t-testing for unequal variances. The critical value for 18 samples and confidence level of $95 \%$ is 2.101 for two-tail and 1.734 for one-tail [13]. Confidence interval is the relationship of sampling error to the parameter of interest [15], [16]. Table 1 displays the tabulated summary of the mean and percent difference of the ECG parameters. The most important parameter, the HR has a percent difference of $0.35 \%$, which falls within the acceptable medical standard of $99 \%$ accuracy.

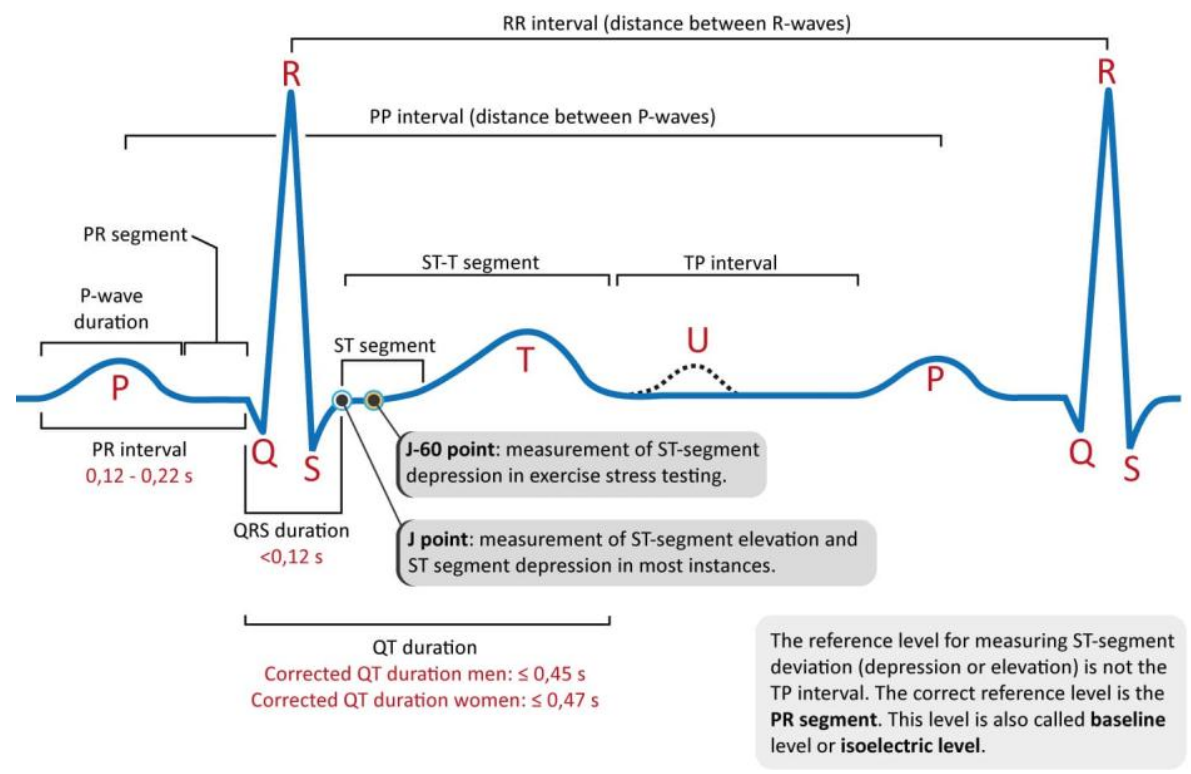

Figure 8. ECG Curve with Its Common Waveform (ECGwaves, 2017) 


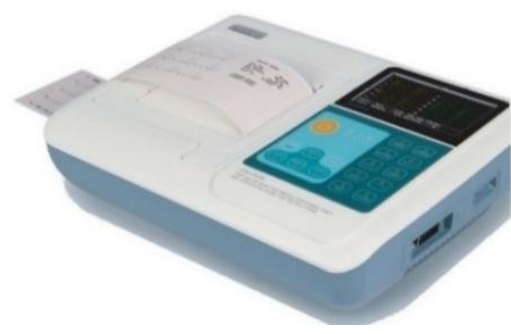

Figure 9. MeCA303i 12 Lead Resting ECG Analysis System (Nova Medical, n.d.)
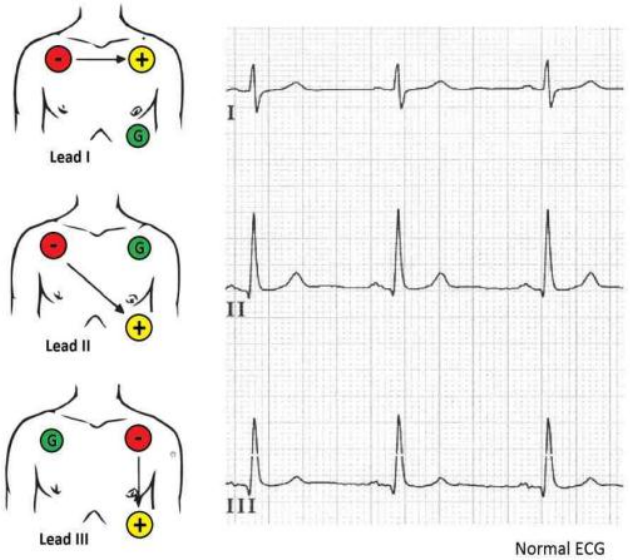

Figure 10 (a). Electrode Placement for Single-Lead ECG Device (Heart Risk Warner, 2015)
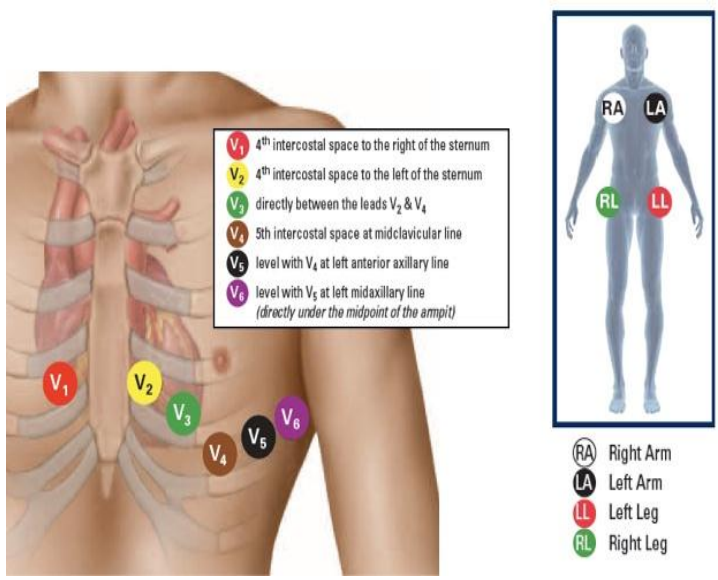

Figure 10 (b). Electrode Placement for Twelve-Lead ECG Device (EMT Resource, 2014)

Table 1. Summary of Mean and Percent Difference of ECG Parameters

\begin{tabular}{cccc}
\hline $\begin{array}{c}\text { ECG } \\
\text { Parameter }\end{array}$ & $\begin{array}{c}\text { 12-Lead ECG } \\
\text { Machine Mean }\end{array}$ & $\begin{array}{c}\text { Single-Lead ECG } \\
\text { Device Mean }\end{array}$ & $\begin{array}{c}\text { Percent Difference } \\
(\%)\end{array}$ \\
\hline PR & 0.1532 & 0.1844 & 18.48 \\
QRS & 0.0959 & 0.1050 & 9.06 \\
QT & 0.3496 & 0.3506 & 0.28 \\
QTc & 0.3977 & 0.3992 & 0.38 \\
PP & 0.7162 & 0.7781 & 8.28 \\
RR & 0.7208 & 0.7782 & 7.66 \\
HR & 78.722 & 79.000 & 0.35 \\
\hline
\end{tabular}

All of the test values, as summarized in Table 2, are less than the critical values, 1.734 and 2.101 for one-tailed and two-tailed tests[13], respectively. The test values fall within the non-critical region. Therefore, the null hypothesis, which states that there is no significant difference between the two sample sets, must not be rejected[13]. In conclusion, there is no significant difference between the samples gathered from the 12lead ECG machine and the samples gathered from the single-lead ECG device prototype.

Table 2. Summary of T-test Value of ECG Parameters

\begin{tabular}{ccc}
\hline ECG Parameter & Two-tailed test value & One-tailed test value \\
\hline PR & 2.032244509 & 1.690924255 \\
QRS & 2.048407142 & 1.701130934 \\
QT & 2.051830516 & 1.703288446 \\
QTc & 2.051830516 & 1.703288446 \\
PP & 2.059538553 & 1.708140761 \\
RR & 2.036933343 & 1.693888748 \\
HR & 2.042272456 & 1.697260887 \\
\hline
\end{tabular}


As shown in Table 3, the percent reliability of the device in detecting ECG conditions is $83.33 \%$. In the 18 test samples gathered from the ECG device prototype, three trials $(3,7,9)$ did not match the Meca3031i status analysis.

Table 3. Tabulated Summary of ECG Status Results Comparison

\begin{tabular}{clc}
\hline Trial & 12-lead ECG Machine & single-lead ECG Device \\
\hline 1 & Normal Sinus Rhythm & Normal Sinus Rhythm \\
2 & Normal Sinus Rhythm & Normal Sinus Rhythm \\
3 & Normal Sinus Rhythm & Sinus Bradycardia \\
4 & Normal Sinus Rhythm & Normal Sinus Rhythm \\
5 & Normal Sinus Rhythm & Normal Sinus Rhythm \\
6 & Normal Sinus Rhythm & Normal Sinus Rhythm \\
7 & Normal Sinus Rhythm & Sinus Tachycardia \\
8 & Normal Sinus Rhythm & Normal Sinus Rhythm \\
9 & Normal Sinus Rhythm & Sinus Tachycardia \\
10 & Normal Sinus Rhythm & Normal Sinus Rhythm \\
11 & Normal Sinus Rhythm & Normal Sinus Rhythm \\
12 & Normal Sinus Rhythm & Normal Sinus Rhythm \\
13 & Normal Sinus Rhythm & Normal Sinus Rhythm \\
14 & Sinus Bradycardia & Sinus Bradycardia \\
15 & Normal Sinus Rhythm & Normal Sinus Rhythm \\
16 & Normal Sinus Rhythm & Normal Sinus Rhythm \\
17 & Normal Sinus Rhythm & Normal Sinus Rhythm \\
18 & Normal Sinus Rhythm & Normal Sinus Rhythm \\
\hline
\end{tabular}

\section{CONCLUSION}

The IoT: Electrocardiogram (ECG) Monitoring System was developed to reduce effort and time overhead, as well as human associated errors during each ward routine visit. A prototype device was developed using a single-lead heart rate monitor sensor front end utilizing 3 ECG electrodes and an Arduino microcontroller. The data gathered from the hardware components are sent to an external computer where it will be processed and analyze by a combination of MATLAB and C\# program. Then, the programs send the collected information into the Internet to be stored in a MySQL database. The information is now viewable in the website. The prototype device was tested against a Meca303i 12-lead ECG machine. Eighteen people were used in the testing. The waveform and heart rate generated from the Meca303i was tested against the device prototype. A comparison of the ECG parameters, PR, QRS, QT, QTc, PP, RR and HR was conducted. The results were analyzed using t-test of sample size $=18$ and $\alpha=0.05$. All parameters gave a test value that lies in the non-critical zone, denoting that the results gathered from the Meca303i 12-lead ECG Machine and the single-lead ECG device prototype possess no significant difference between each other. The device's capability to determine ECG status results to $83.33 \%$ reliability. A $0.35 \%$ difference in the heart rate is shown. Statistics indicates that the device is functioning well as an alternative to an actual ECG machine.

\section{ACKNOWLEDGEMENTS}

This work has been supported by the Computer Engineering Department, Malayan Colleges Laguna, Pulo Diezmo Rd., Cabuyao City, Laguna, 4025 Philippines.

\section{REFERENCES}

[1] P. Jevon and B. Ewens, Monitoring the critically ill patient: John Wiley \& Sons, 2012.

[2] C. M. Jadhav and V. Bairagi, "Detection \& Classification of Cardiac Arrhythmia," International Journal of Informatics and Communication Technology (IJ-ICT), vol. 6, pp. 31-36, 2017.

[3] G. Christopher, "Internet of Things in Healthcare: What's Next for IoT Technology in the Health Sector," ed, 2016.

[4] M. Abo-Zahhad, S. M. Ahmed, and O. Elnahas, "A wireless emergency telemedicine system for patients monitoring and diagnosis," International journal of telemedicine and applications, vol. 2014, p. 4, 2014.

[5] M. S. Patil, M. S. Jagdale, M. A. G. A. P. Ms, and R. Tapase, "WIRELESS ECG MONITORING SYSTEM," 2016.

[6] J. A. Walsh, E. J. Topol, and S. R. Steinhubl, "Novel wireless devices for cardiac monitoring," Circulation, vol. 130, pp. 573-581, 2014.

[7] F. Miao, Y. Cheng, Y. He, Q. He, and Y. Li, "A wearable context-aware ECG monitoring system integrated with built-in kinematic sensors of the smartphone," Sensors, vol. 15, pp. 11465-11484, 2015.

[8] B. G. Irianto, B. Budhiaji, and S. Syaifudin, "Design of Electro Cardiograph Machine Based on Atmega Microcontroller," Indonesian Journal of Electrical Engineering and Computer Science, vol. 2, pp. 328-333, 2016. 
[9] A. Rizal, V. Suryani, J. Jondri, and S. Hadiyoso, "Development of Wireless Patient's Vital Sign Monitor Using Wireless LAN (IEEE. 802.11. b/g) Protocol," International Journal of Electrical and Computer Engineering, vol. 4, p. 893, 2014.

[10] N. M. N. Daud, N. A. A. A. Bakar, and H. M. Rusli, "Implementing rapid application development (RAD) methodology in developing practical training application system," in Information Technology (ITSim), 2010 International Symposium in, 2010, pp. 1664-1667.

[11] G. Fent, J. Gosai, and M. Purva, "Teaching the interpretation of electrocardiograms: Which method is best?," Journal of electrocardiology, vol. 48, pp. 190-193, 2015.

[12] P. H. Charlton, T. Bonnici, L. Tarassenko, J. Alastruey, D. A. Clifton, R. Beale, et al., "Extraction of respiratory signals from the electrocardiogram and photoplethysmogram: technical and physiological determinants," Physiological Measurement, vol. 38, p. 669, 2017.

[13] T. Greenfield, "Ethics of research," Research Methods for Postgraduates, p. 46, 2016

[14] M. G. Khan, Rapid ECG interpretation: Springer Science \& Business Media, 2008.

[15] J. Frost. (2013). When Should I Use Confidence Intervals, Prediction Intervals, and Tolerance Intervals. Available: http://blog.minitab.com/blog/adventures-in-statistics-2/when-should-i-use-confidence-intervals-predictionintervals-and-tolerance-intervals

[16] M. Weippert, M. Kumar, S. Kreuzfeld, D. Arndt, A. Rieger, and R. Stoll, "Comparison of three mobile devices for measuring R-R intervals and heart rate variability: Polar S810i, Suunto t6 and an ambulatory ECG system," European journal of applied physiology, vol. 109, pp. 779-786, 2010. 


\section{BIOGRAPHIES OF AUTHORS}

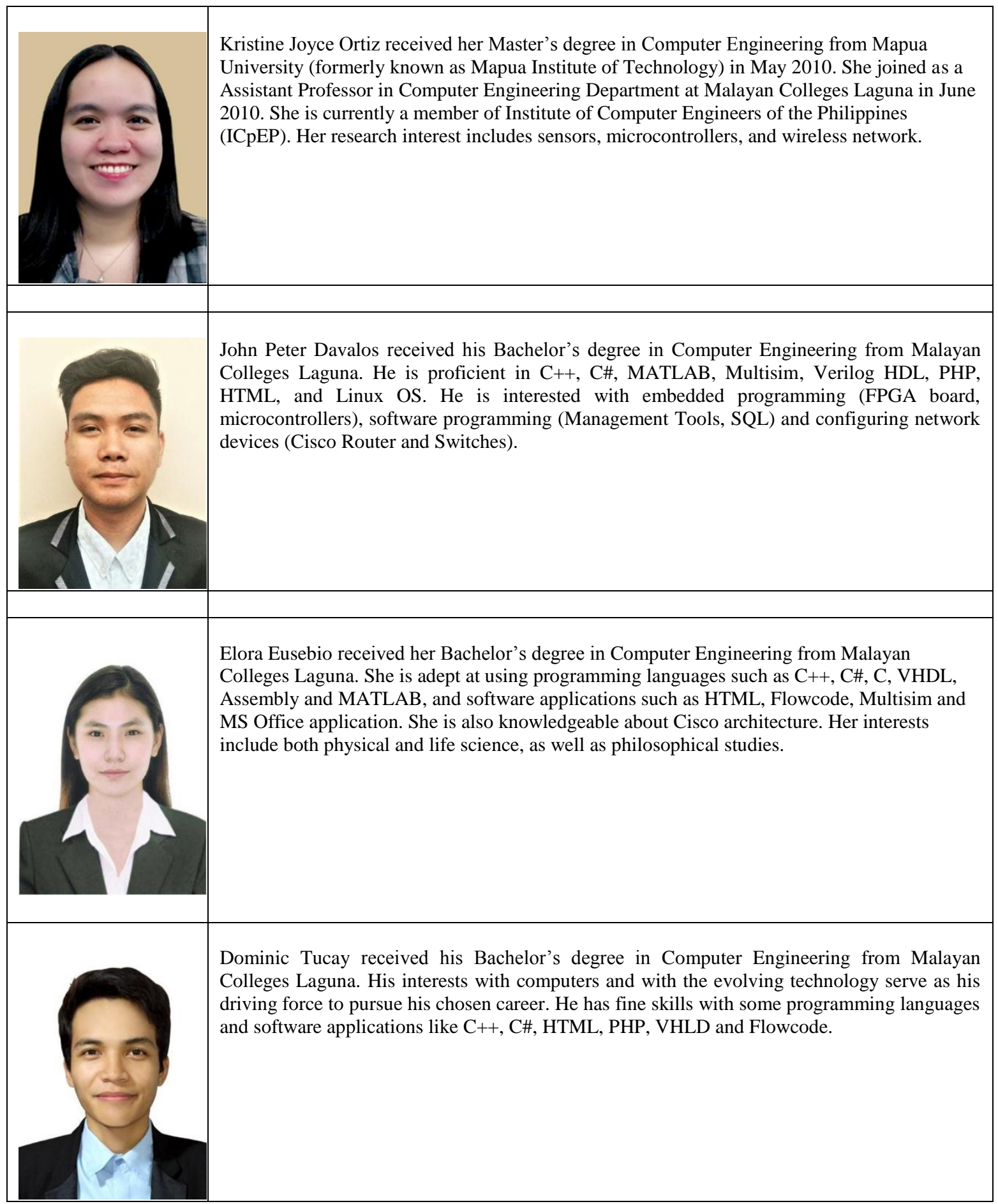

